\title{
On the Effects of Modeling As-Manufactured Geometry: Toward Digital Twin
}

\author{
Albert Cerrone, ${ }^{1}$ Jacob Hochhalter, ${ }^{2}$ Gerd Heber, ${ }^{3}$ and Anthony Ingraffea ${ }^{4}$ \\ ${ }^{1}$ School of Civil and Environmental Engineering, Cornell University, 642 Rhodes Hall, Ithaca, NY 14853, USA \\ ${ }^{2}$ NASA Langley Research Center, 188E, Hampton, VA 23681, USA \\ ${ }^{3}$ The HDF Group, 1800 South Oak Street, Suite 203, Champaign, IL 61820, USA \\ ${ }^{4}$ School of Civil and Environmental Engineering, Cornell University, 643 Rhodes Hall, Ithaca, NY 14853, USA
}

Correspondence should be addressed to Albert Cerrone; arc247@cornell.edu

Received 23 April 2014; Revised 26 July 2014; Accepted 1 August 2014; Published 27 August 2014

Academic Editor: Christopher J. Damaren

Copyright (C) 2014 Albert Cerrone et al. This is an open access article distributed under the Creative Commons Attribution License, which permits unrestricted use, distribution, and reproduction in any medium, provided the original work is properly cited.

\begin{abstract}
A simple, nonstandardized material test specimen, which fails along one of two different likely crack paths, is considered herein. The result of deviations in geometry on the order of tenths of a millimeter, this ambiguity in crack path motivates the consideration of asmanufactured component geometry in the design, assessment, and certification of structural systems. Herein, finite element models of as-manufactured specimens are generated and subsequently analyzed to resolve the crack-path ambiguity. The consequence and benefit of such a "personalized" methodology is the prediction of a crack path for each specimen based on its as-manufactured geometry, rather than a distribution of possible specimen geometries or nominal geometry. The consideration of as-manufactured characteristics is central to the Digital Twin concept. Therefore, this work is also intended to motivate its development.
\end{abstract}

\section{Introduction}

Current structural life-management approaches consider a variety of sources of uncertainty in producing reliability estimates. Typically, an empirical worst-case scenario is considered for design and scheduling inspections. However, relying on the worst-case scenario seen during testing assumes that in-service loading conditions are well understood, the tails of distributions of material behavior are accurately modeled, and all coupled damage modes that lead to reduced life are accounted for during testing. During service, decisions regarding the capability of a structure to endure a mission are based on these uncertainty and reliability estimates, along with relatively basic information of initial design specifications, usage history, and nondestructive evaluation (NDE) and maintenance records. Unfortunately, without a detailed record of vehicle-specific usage, variability in usage across a fleet only adds to the uncertainty in the state of a particular vehicle. Furthermore, this assumption of representative, worst-case conditions leads to costly inspection or replacement of parts which likely contain acceptable damage [1].

By Bayesian inference, it is possible to combine new usage data with existing predictions to improve reliability estimates throughout the service life of a vehicle. Such predictions are practical if vehicle-specific initial and updated state, usage history, and NDE findings are recorded throughout the service life and subsequently used for updated prognoses. This is because no two structural components within a fleet are equivalent in as-manufactured geometry or material microstructure, and no two vehicles experience equivalent usage or environment during their lifetime.

Digital Twin [2,3] is an emerging management and certification method designed to address these existing issues; however, this paper does not detail Digital Twin in its entirety-topics such as multiscale and multiphysics modeling, model integration, and computational demands are beyond the scope of this work. This paper focuses on the efficacy of modeling and simulating the as-manufactured geometry of each individual component in a fleet and aims to 


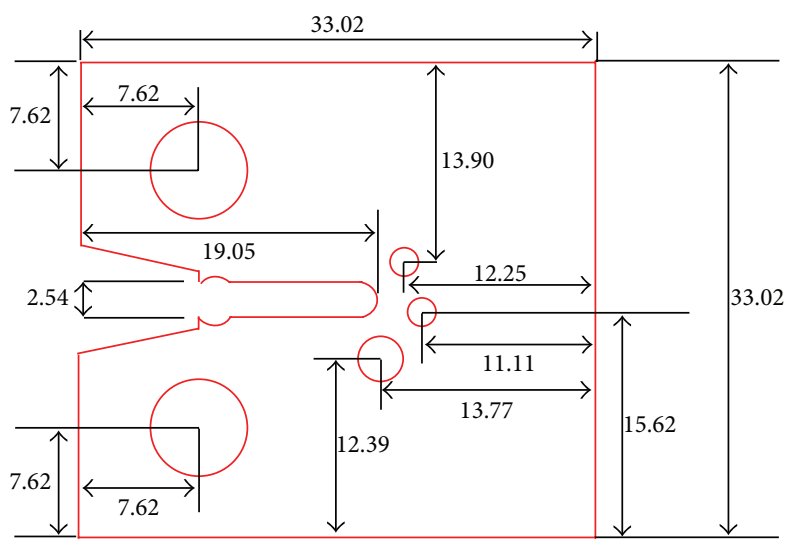

(a)

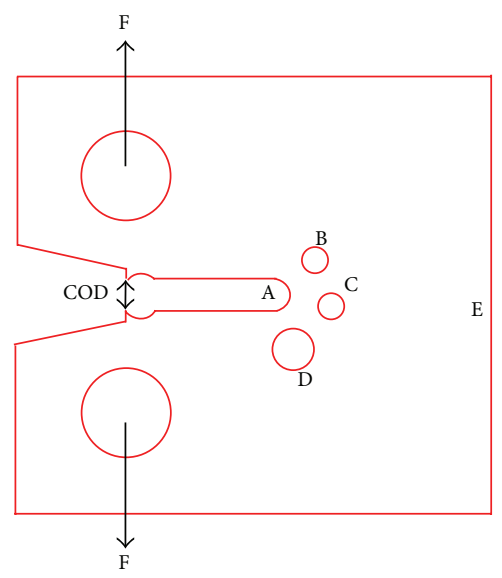

(b)

FIGURE 1: Nominal dimensions of the challenge specimen with $3.18 \mathrm{~mm}$ nominal thickness. All dimensions are in millimeters. Figure was adapted from the SFC lead article [4].

both motivate and better define Digital Twin with a straightforward use case. The presented use case, ductile fracture in a nonstandardized material test specimen, dispels the notion that modeling damage in a component can be done with nothing but a representative geometry, a seemingly well-calibrated constitutive model, and sound engineering judgment. A degree of personalization is required, the effectiveness of which is demonstrated by considering the 2012 Sandia Fracture Challenge (SFC) [4]. Specifically, the nonstandardized SFC geometry was observed to fail along one of two crack paths. A result of deviations in geometry on the order of tenths of a millimeter, this crack path ambiguity suggests that the specimen does not have a single representative geometry and motivates modeling the asmanufactured components, a cornerstone of Digital Twin.

This paper is divided into three parts. In the first, Section 2, the 2012 Sandia Fracture Challenge is introduced and outlined in light of the aforementioned crack path ambiguity. The primary outcome conveyed in this section is that no single configuration adequately represents the SFC geometry. Consequently, some modicum of personalization must be injected into the SFC-related modeling activity. In the second part, Sections 3 and 4, digital twin specimens are modeled and simulated in a commercial finite element software package, Abaqus/Explicit, to resolve crack path ambiguity in the SFC geometry. The computational model is refined during the course of the study with the introduction of the as-manufactured specimen geometry and the accommodation of the shear damage mode, thereby mimicking some of the data acquisition and updating procedures within Digital Twin. In the third part, Section 5, the shift to a higher fidelity computational model is presented in the context of relevant experimental results. Hereafter, Digital Twin refers to the paradigm and digital twin refers to an instantiation.

\section{The 2012 Sandia Fracture Challenge}

2.1. Overview. The Sandia Fracture Challenge (SFC) was issued by Sandia National Laboratories in partnership with the National Science Foundation (NSF) and Naval Surface Warfare Center Carderock Division in 2012 to predict crack initiation and propagation in a novel geometry, the "challenge specimen" hereafter. For details regarding the experiments and the round robin predictions made by the thirteen participants, the reader is directed to the SFC lead article [4]. This section serves as a brief summary of the experimental setup, the challenge requirements, relevant experimental outcomes, and the participants' approaches.

2.2. Experimental Setup. The material of interest was an offthe-shelf alloy, 15-5 PH: a precipitation hardened stainless steel. Thirteen challenge specimens as well as tensile and fracture toughness test specimens were machined from the same plate. It is noteworthy that the challenge specimens were ordered with a $0.0508 \mathrm{~mm}$ tolerance on all dimensions; however, not all of the specimens were machined to specifications, making for the peculiar experimental results detailed in Section 2.4. The nominal dimensions of the challenge specimen are given in Figure 1.

The challenge specimen has several similarities with a standard compact tension (CT) fracture toughness specimen, but has three holes beyond the blunt notch. Following Figure 1(a), a labeling convention is used to identify pertinent features of the specimen: blunt notch (A), $3.05 \mathrm{~mm}$-diameter hole (D), $1.78 \mathrm{~mm}$-diameter hole beyond blunt notch (C), $1.78 \mathrm{~mm}$-diameter hole above holes $\mathrm{C}$ and $\mathrm{D}(\mathrm{B})$, and the midpoint of the far-right edge (E). Each specimen was loaded at the pin holes at a rate of $0.0127 \mathrm{~mm} / \mathrm{s}$. All tests were conducted at lab ambient temperature.

2.3. Quantities of Interest. Each team was required to predict six quantities of interest (QOIs) identified by Sandia. They were

(1) crack path,

(2) load versus crack opening displacement (COD), Figure 1, 
(3) load when a crack first initiated,

(4) COD when a crack first initiated,

(5) load when a crack later reinitiated from a second feature (i.e., hole B or C),

(6) COD when a crack later reinitiated from a second feature (i.e., hole B or C).

2.4. Experimental Outcomes: Crack Path Ambiguity. Two crack paths were observed in the challenge specimens: A$\mathrm{C}-\mathrm{E}$ and A-D-C-E. Of the thirteen specimens, ten cracked along the path defined by A-D-C-E (D2, S1, S2, S3, S4, S5, S6, S7, S8, and S11) while three cracked along A-C-E (D1, S9, and S10). It is noteworthy that only one of the specimens was machined to specifications and its crack path was A-C-E; the others exhibited deviations beyond the $0.0508 \mathrm{~mm}$ machine tolerance ranging from a few microns to twice the tolerance. After extensive analysis, Boyce et al. [4] determined that these deviations from the nominal dimensions were the root cause of the ambiguity in crack path.

In particular, the location of hole $\mathrm{C}$ in relation to hole D was decisive. Specimens with holes C and D separated by $2.381 \mathrm{~mm}$ or less (measured here as the vertical distance between the tops of holes $\mathrm{C}$ and $\mathrm{D}$ ) consistently cracked along A-D-C-E. Conversely, specimens whose crack path was A-CE exhibited distances between holes C and D of $2.401 \mathrm{~mm}$, $2.393 \mathrm{~mm}$, and $2.397 \mathrm{~mm}$. An outlier specimen, S11, had holes $\mathrm{C}$ and $\mathrm{D}$ separated by a distance of $2.398 \mathrm{~mm}$ but cracked along A-D-C-E. To rationalize this outlier, Boyce et al. [4] considered the ratio of the vertical distance between the blunt notch $(\mathrm{A})$ and hole $\mathrm{D}$ to the horizontal distance between the blunt notch $(\mathrm{A})$ and hole $\mathrm{C}$ and determined that this ratio for the S11 specimen, along with the other specimens' ratios which cracked along A-D-C-E, was greater than machine tolerance.

The load versus COD profiles for the thirteen specimens is given in Figure 2. Perhaps most obvious from the profiles is that specimens that cracked along A-C-E had a considerably longer delay in the first load drop than specimens that cracked along A-D-C-E. The overall load drop from A to $\mathrm{C}$ for all specimens was roughly $3 \mathrm{kN}$, but the change in total COD for specimens that cracked along A-D-C-E during this reduction was greater than that of specimens that cracked along A-C-E.

2.5. Survey of Participants' Predictive Capabilities. An extensive array of capabilities was employed to predict the six QOIs. Several teams used porous metal plasticity (void growth) models [6]. Others employed von Mises plasticity supplemented by a fracture model (i.e., Mohr-Coulomb [7] and Johnson-Cook [5]). The extended finite element method (XFEM) [8], material point method, peridynamic theory, and cohesive zones were also employed. Some of these methods outperformed others in determining some of the QOIs, but no single model accurately addressed all of the QOIs. The following section details one of these models: a commercially available, porous metal plasticity-based approach.

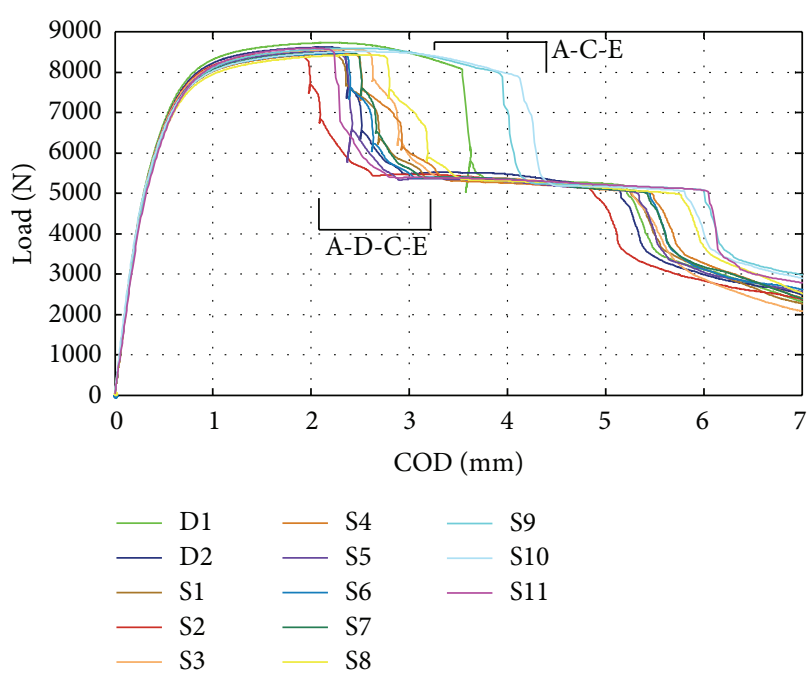

Figure 2: Load versus COD measurements for thirteen challenge specimens $[4,5]$.

\section{Computational Model}

3.1. Material Model and Calibration. The porous metal plasticity model implemented in Abaqus/Explicit [9] is a voidgrowth plasticity model given by Gurson [10]. Both the elastic response and hardening behavior are isotropic. The yield condition is given by

$$
\Phi=\frac{\sigma_{e}^{2}}{\sigma_{M}^{2}}+2 f \cosh \left(\frac{\sigma_{k}^{k}}{2 \sigma_{M}}\right)-\left(1+f^{2}\right)=0,
$$

where $f$ is void volume fraction, $\sigma_{e}$ is the effective Mises stress, $\sigma_{M}$ is the yield stress of the fully dense matrix, and $\sigma_{k}^{k}$ is three-times the hydrostatic stress. Tvergaard [11] modified (1) to include yield surface parameters $q_{1}, q_{2}$, and $q_{3}$ :

$$
\Phi=\frac{\sigma_{e}^{2}}{\sigma_{M}^{2}}+2 f q_{1} \cosh \left(\frac{q_{2} \sigma_{k}^{k}}{2 \sigma_{M}}\right)-\left(1+q_{3} f^{2}\right)=0,
$$

where Gurson's yield condition is recovered for $q_{1}=q_{2}=$ $q_{3}=1$. Damage is introduced into the model by way of void coalescence. Two material parameters control the damage process: critical void volume fraction, $f_{c}$, and void volume fraction at total failure, $f_{f}$. If the void volume fraction exceeds $f_{f}$, a material point loses all capacity to carry stress. Moreover, if all of an element's material points fail, the element is removed from the discretization. If a material point's void volume fraction falls between $f_{c}$ and $f_{f}, f$ in (2) is replaced by the expression

$$
f_{c}+\frac{\left(\left(q_{1}+\sqrt{q_{1}^{2}-q_{3}}\right) / q_{3}\right)-f_{c}}{f_{f}-f_{c}}\left(f-f_{c}\right) .
$$

The Gurson model with Tvergaard's modification was calibrated against both the 15-5 $\mathrm{PH}$ tensile and fracture toughness test data detailed in the SFC lead article [4]. The test geometries were generated in Abaqus/CAE and meshed 
TABLE 1: Calibrated porous metal plasticity parameters.

\begin{tabular}{lc}
\hline Parameter & Value \\
\hline Mass density & $7.8 \mathrm{~g} / \mathrm{mm}^{3}$ \\
$E$ & $235.4 \mathrm{GPa}$ \\
$\nu$ & 0.272 \\
$r$ & 0.99 \\
$q_{1}, q_{2}, q_{3}$ & $1,1,1$ \\
$\varepsilon_{N}$ & $0.09 \mathrm{~mm} / \mathrm{mm}$ \\
$s_{N}$ & $0.001 \mathrm{~mm} / \mathrm{mm}$ \\
$f_{N}$ & 0.01 \\
$f_{f}$ & 0.18 \\
$f_{c}$ & 0.10 \\
\hline
\end{tabular}

with $200 \mu \mathrm{m}$-sized quadratic tetrahedral elements. The calibration was conducted in two stages. In the first, several candidate sets of parameters were identified that reproduced the experimental stress-strain tensile data. Thereafter, the candidates that did not accurately reproduce the reduction in cross-sectional area observed during the tensile tests were discarded. In the second stage, the fracture toughness test was simulated with the remaining candidates, and the set that most accurately reproduced the measured force versus COD was retained for the production-level challenge specimen simulations. This set is given in Tables 1 and 2 .

3.2. Mesh Refinement and Sensitivity. It is well established that solutions from porous metal plasticity models are mesh sensitive. Mesh refinement studies were conducted on the challenge specimen with the nominal specimen geometry. Nominal specimen dimensions were used initially since no as-manufactured geometry was provided. $200 \mu \mathrm{m}$-sized tetrahedral elements were placed beyond the blunt notch (A) and around the holes B, C, and D because this element size was used during calibration. As shown in Figure 3, depending on the extent of these $200 \mu \mathrm{m}$-sized elements beyond the holes, the crack was predicted to propagate along one of two paths, A-C-E or A-D-C-E. In cases where the mesh was coarsened to the back edge, $\mathrm{E}$, the predicted crack path was A-D-C-E; however, in cases where the $200 \mu \mathrm{m}$-sized elements were retained out to $\mathrm{E}$, the predicted crack path was A-CE. The latter result is in agreement with experimentationthe one specimen machined to specifications (i.e., within $0.0508 \mathrm{~mm}$ of nominal) cracked along A-C-E. Consequently, for all production-level simulations with the challenge specimen, the $200 \mu \mathrm{m}$-sized tetrahedral elements were retained up to $\mathrm{E}$.

\subsection{Challenge Specimen's Boundary Conditions and Compu-} tational Demands. The loading pins' action on the specimen was modeled with kinematic coupling constraints. The loading pin closest to hole B was held stationary while the other was gradually displaced downwards $7.5 \mathrm{~mm}$. A kinematic boundary condition was assigned to a single node near $\mathrm{E}$ to restrict rigid body rotation.

All production-level runs had approximately 5.5 million degrees of freedom. Simulations were conducted on a $3.40 \mathrm{GHz}$, 4th generation Intel Core i7 processor.
Abaqus/Explicit's shared memory parallelization on four threads with a targeted time increment of $1 e-6$ seconds resulted in approximately a 4 -day wall-clock run time. However, explicit integration of a finite element model scales well since the global stiffness matrix is not formed (and therefore, also not inverted) and the mass matrix can be diagonalized, resulting in a system of uncoupled equations. Therefore, it is expected that the simulation time could be significantly reduced for codes where the number of employed CPUs at runtime is not limited by the number of available software licenses.

\section{Numerical Simulations and Results}

4.1. Overview. The computational model was initially employed to predict fracture in the challenge specimen. Since no as-manufactured geometries were provided initially, the prediction was conditioned on a single (nominal) geometry, Section 4.2. After the blind prediction had been made, as-manufactured configurations were considered for probing the model's ability to resolve the ambiguity in crack path, Sections 4.2-4.4. Also, based on the experimental results, the model was updated with new porous metal plasticity model parameters to improve its predictive capabilities, Section 4.5, emulating a Bayesian update to the digital twin.

4.2. Modeling the Challenge Specimen with Nominal Dimensions. The challenge specimen with the nominal dimensions given in Figure 1 was modeled for determining a baseline simulated crack path and load versus COD profile. The predicted crack path was A-C-E, Figure 4. The predicted peak load and magnitude of the first load drop agreed with the profile of $\mathrm{D} 1$, the only specimen manufactured to specifications; however, the predicted $0.8 \mathrm{~mm}$ change in total COD during the first load drop was far greater than that of any of the thirteen specimens.

As with the nominal specimen, the simulation of the D1 digital twin specimen predicted an A-C-E crack path, matching the experiment, Figure 5. The predicted peak load was only $2.2 \%$ lower than that measured during the experiment. Moreover, the simulation predicted the magnitude of the first load drop relatively accurately; however, the predicted $1.0 \mathrm{~mm}$ change in total COD during the first load drop was far greater than that observed. The crack's initiation from hole $\mathrm{C}$ was not captured in the simulation; due to excessive element deformation, a converged solution could not be reached and the simulation subsequently terminated. This was not the result of physical phenomena, rather the violation of the Courant-Friedrichs-Lewy (CFL) condition [12]. Moreover, due to limitations with Abaqus/Explicit, new elements could not be adaptively inserted into the discretization to circumvent this issue.

4.3. Modeling the S5 (out of Specifications) Specimen. The S5 specimen, one exhibiting some of the largest deviations from specifications, was one of ten in which the crack propagated along the A-D-C-E path. With regards to the aforementioned ratio of the vertical distance between the blunt notch (A) and 
TABLE 2: Parameters for tabular hardening curve.

\begin{tabular}{lccccc}
\hline Plastic strain $(\mathrm{mm} / \mathrm{mm})$ & Yield stress $(\mathrm{MPa})$ & Plastic strain $(\mathrm{mm} / \mathrm{mm})$ & Yield stress $(\mathrm{MPa})$ & Plastic strain $(\mathrm{mm} / \mathrm{mm})$ & Yield stress $(\mathrm{MPa})$ \\
\hline 0.0 & 1092.5 & 0.05111 & 1181.7 & 0.12 & 1250.0 \\
0.01649 & 1127.6 & 0.06852 & 1198.5 & 0.16 & 1320.0 \\
0.03404 & 1156.7 & 0.07429 & 1200.0 & 1.0 & 1350.0 \\
\hline
\end{tabular}
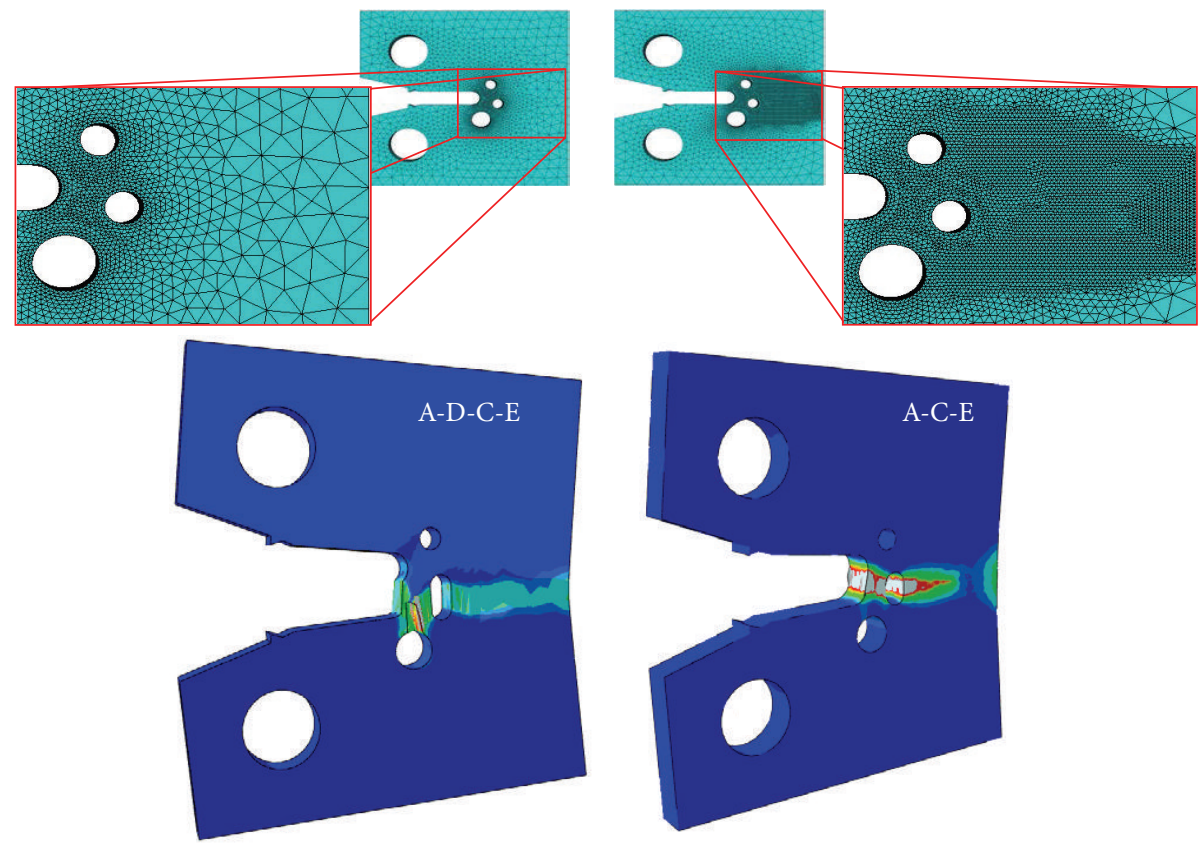

Figure 3: Influence of mesh refinement on predicted crack path.

hole $\mathrm{D}$ to the horizontal distance between the blunt notch (A) and hole $\mathrm{C}$, the S5 specimen exhibited the largest deviation from nominal.

The simulation of the S5 digital twin specimen reproduced the A-D-C-E crack path, Figure 6. The predicted peak load corresponded to the experimental peak. As with the D1 specimen, the magnitudes of the simulated and experimental load drops were nearly indistinguishable; however, the predicted $1.7 \mathrm{~mm}$ change in total COD during the first load drop was far greater than the observed $0.5 \mathrm{~mm}$.

4.4. Modeling the S11 (Slightly out of Specifications) Specimen. The S11 specimen, one exhibiting slight deviations from specifications, was one of ten that cracked along A-D-CE. This result was somewhat peculiar because the spacing between holes $\mathrm{C}$ and $\mathrm{D}$ in the S11 specimen was characteristic of specimens which cracked along A-C-E. As mentioned earlier, its ratio of the vertical distance between $\mathrm{A}$ and $\mathrm{D}$ to the horizontal distance between $\mathrm{A}$ and $\mathrm{C}$ was greater than machine tolerance (a characteristic of all specimens failing A-D-C-E), and this is perhaps the best, and only, geometricbased explanation as to why the S11 specimen cracked along A-D-C-E.

The simulation of the S11 digital twin specimen did not reproduce the A-D-C-E crack path, Figure 7. As with the simulations discussed previously, the peak load was accurately predicted and the simulated change in total COD during the first load drop was significantly higher than the measured peak. To shed light on the inaccurate prediction of crack path, additional analyses were conducted. Hole $\mathrm{C}$ was shifted incrementally closer to hole $\mathrm{D}$ until the A-D-C-E crack path was predicted. A $50 \mu \mathrm{m}$ downward vertical translation of hole $\mathrm{C}$ was not enough to yield an A-D-C-E path; however, as shown in Figure 7, compared to the simulation of the S11 specimen, the void volume fraction between the blunt notch $A$ and hole $C$ significantly declined while it increased between the blunt notch $\mathrm{A}$ and hole $\mathrm{D}$, indicating a stronger propensity for crack growth into hole D. A subsequent $10 \mu \mathrm{m}$ translation (making for a total translation of $60 \mu \mathrm{m}$ ) resulted in the A-DC-E crack path.

With regards to the predicted load versus COD profiles, the two simulations showing A-C-E had relatively similar curves. A smaller COD was required to initiate the first load drop in the S11 specimen compared to D1 and S5an expected result as a significant amount of COD went into plastically deforming the ligaments $\mathrm{A}-\mathrm{D}$ and $\mathrm{A}-\mathrm{C}$ in the other model. The simulation with the $60 \mu \mathrm{m}$ translation had problems converging due to excessive element deformation as the ligament A-D was beginning to fail, again the result of violating the CFL condition.

4.5. Updating the Computational Model. Although modeling the as-manufactured geometry improved crack path predictions, the first iteration of the framework did a poor job of 


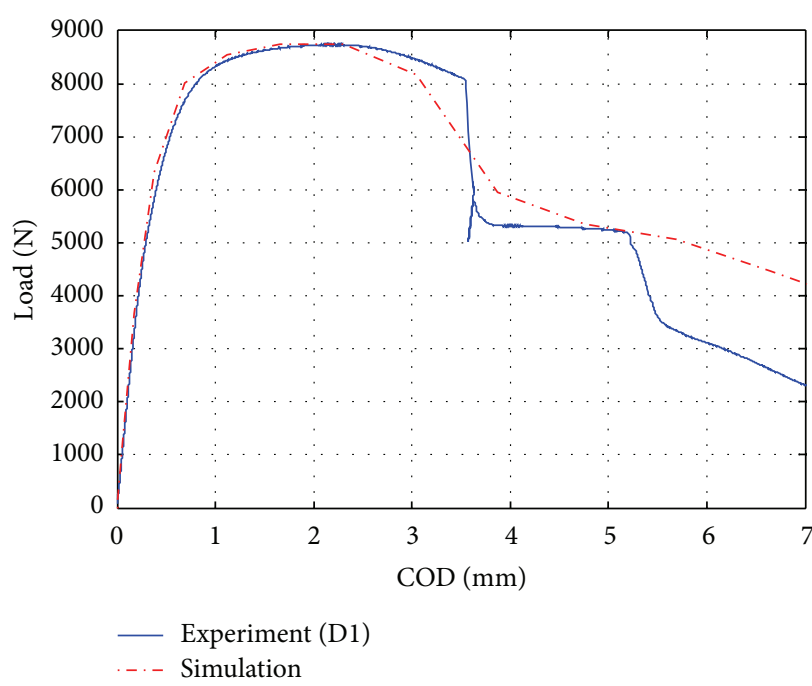

(a)
Void volume

fraction
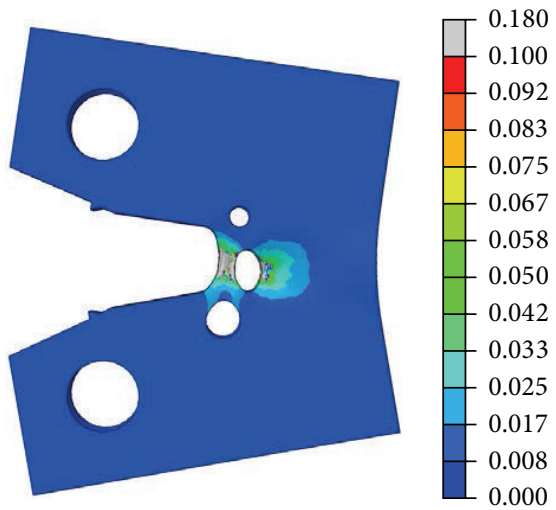

(b)

FIGURE 4: Experimental load versus COD profiles for D1 specimen and simulated load verus COD profile for nominal specimen, (a). The contour of void volume fraction, showing void accumulation along ligament $\mathrm{A}-\mathrm{C}$, taken at load $=4,471 \mathrm{~N}$, (b).

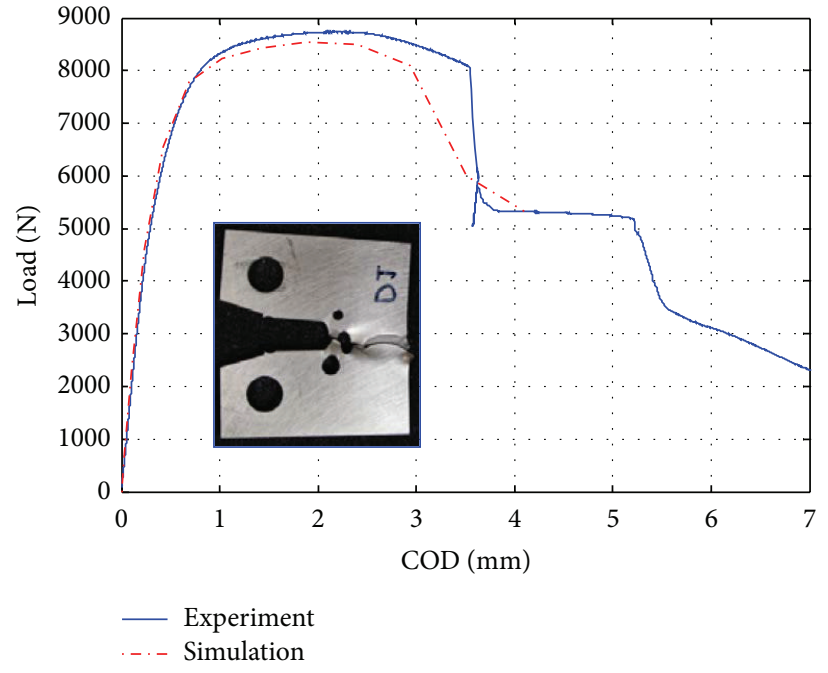

(a)

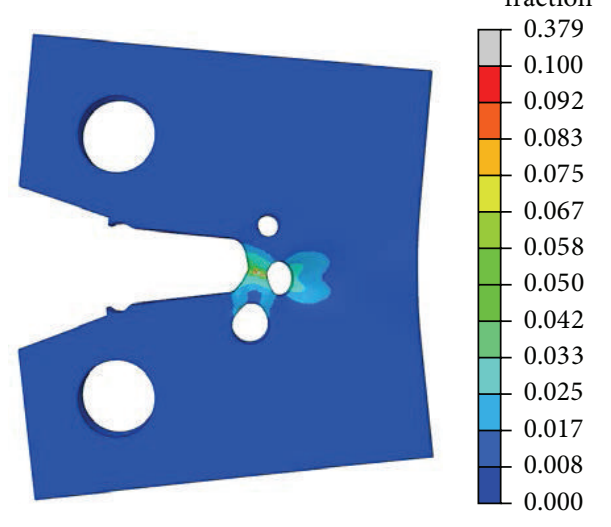

(b)

FIGURE 5: Experimental and simulated load versus COD profiles for D1 specimen, (a). The contour of void volume fraction, showing void accumulation along ligament A-C, taken at load = 5,333 N, (b). Photograph of specimen adapted from [4].

capturing the immediacy of the first load drop. This is most evident for the S5 and S11 digital twin specimens which failed along A-D-C-E. Material points along the critical ligaments simply did not fail soon enough nor at a fast enough rate. It is well known that the Gurson model performs poorly in sheardominated regimes. Specifically, the model underpredicts damage under conditions of low stress triaxiality. Note that stress triaxiality is the ratio between hydrostatic and von Mises stresses. The A-D-C-E case had an unexpected shear damage mode, invalidating the use of the unmodified Gurson model. To update the digital twin models, this unexpected damage mode must now be accommodated. Nahshon and
Hutchinson [13] proposed a modification to Gurson's model to compensate for the aforementioned limitation; specifically, they consider a two-term void evolution law wherein $f$ is increased under shear deformation through the intercession of $J_{3}$, the third invariant or determinant of the deviatoric stress tensor. Nahshon et al. [6] employed this modification to model fracture in the S5 specimen, and their prediction was considerably more accurate as a result.

To compensate for the Gurson model's inability to evolve damage under low stress triaxiality, a user subroutine was employed to track stress triaxiality in real-time. When a material point had stress triaxiality greater than or equal to 


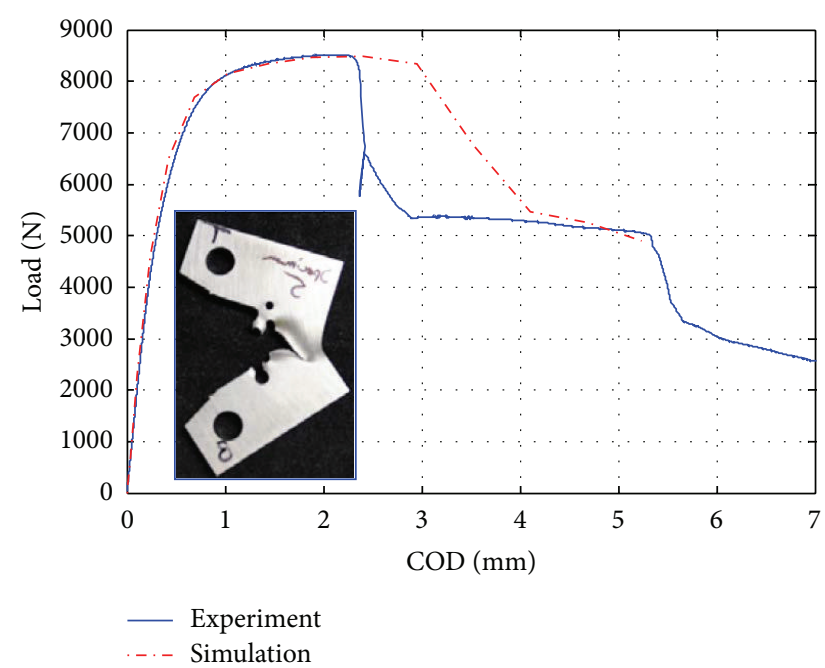

(a)

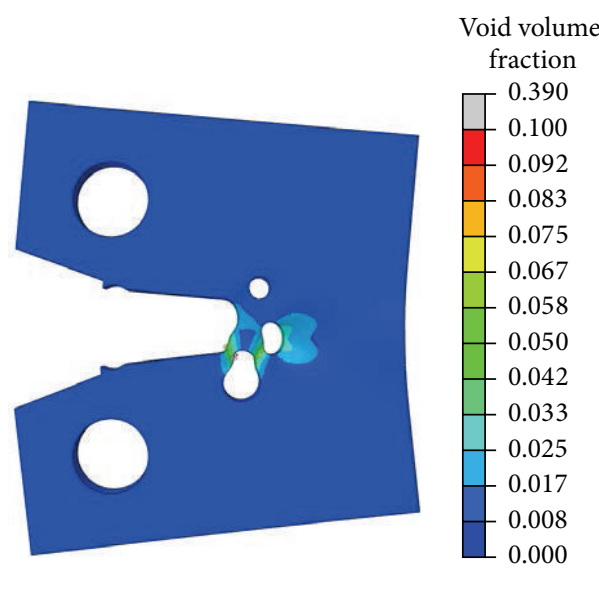

(b)

FIGURE 6: Experimental and simulated load versus COD profiles for S5 specimen, (a). The contour of void volume fraction, showing void accumulation along ligaments A-D and D-C, taken at load = 5,474 N, (b). Photograph of specimen adapted from [4].

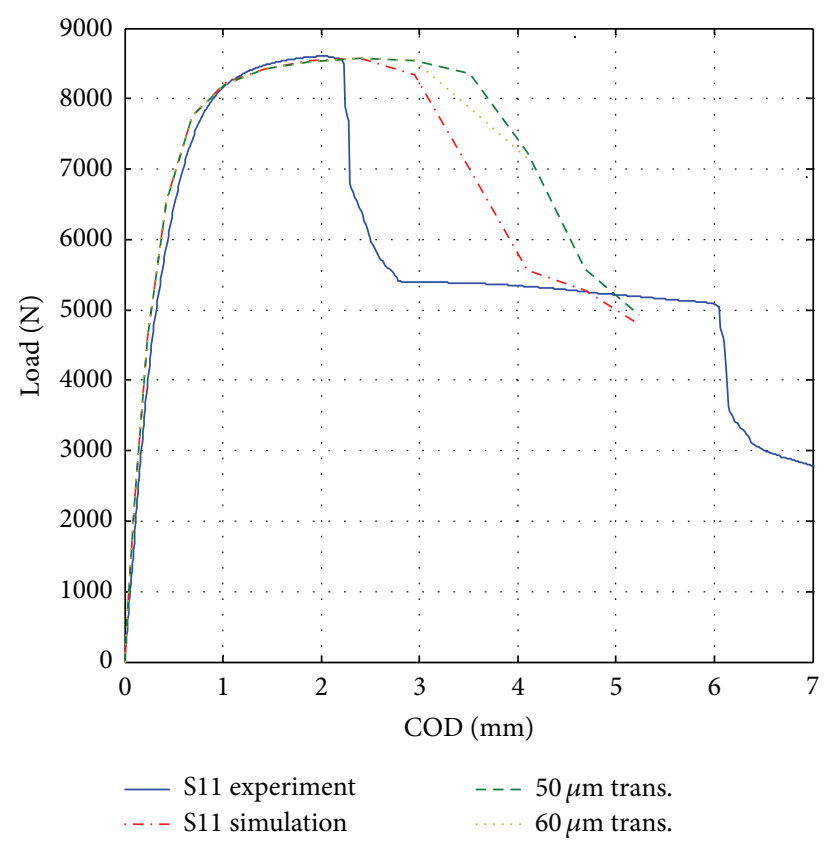

(a)

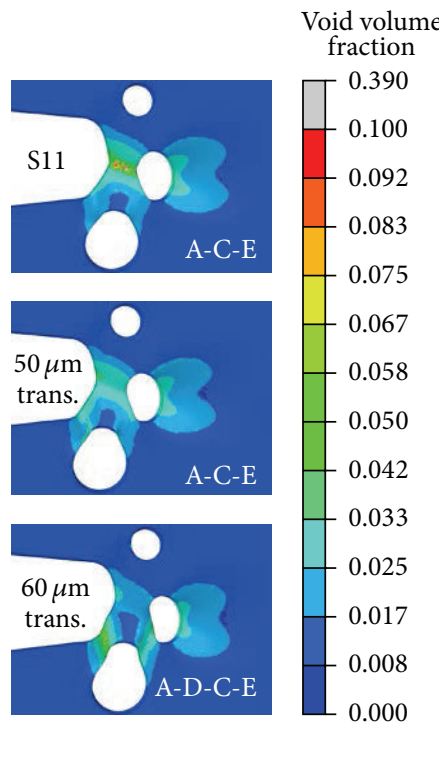

(b)

FIGURE 7: Experimental S11 load versus COD profile, simulated S11 load versus COD profile, simulated S11 with hole C translated down $50 \mu \mathrm{m}$ load versus COD profile, and simulated S11 with hole C translated down $60 \mu \mathrm{m}$ load versus COD profile, (a). The contours of void volume fraction were taken at load $=5,528 \mathrm{~N}, 7,239 \mathrm{~N}$, and 7,140 N for S11, S11 w/50 $\mu \mathrm{m}$ translation, and S11 w/60 $\mu \mathrm{m}$ translation, respectively, (b).

some value $t$, its yield surface parameters were set to $q_{1}=1.5$, $q_{2}=1.0$, and $q_{3}=q_{1}^{2}=2.25$ (values commonly applied to steel in the literature). If a material point had a stress triaxiality lower than $t$, its $q_{2}$ was set to 2.0 to increase the second term in the yield condition given in (2) (effectively inducing damage). This methodology was adopted from Chabanet et al. [14] who used it to model crack growth in aluminum sheets.
This scheme was implemented for the S5 digital twin specimen. The improvements made over the previous iteration's predictions were significant, suggesting that stress triaxiality should not be ignored when modeling challenge specimens that failed A-D-C-E. First, the damage rate in ligament A-D was accelerated. This caused a more immediate first load drop. Second, the first load drop was predicted to initiate at a much lower COD, in stronger agreement 


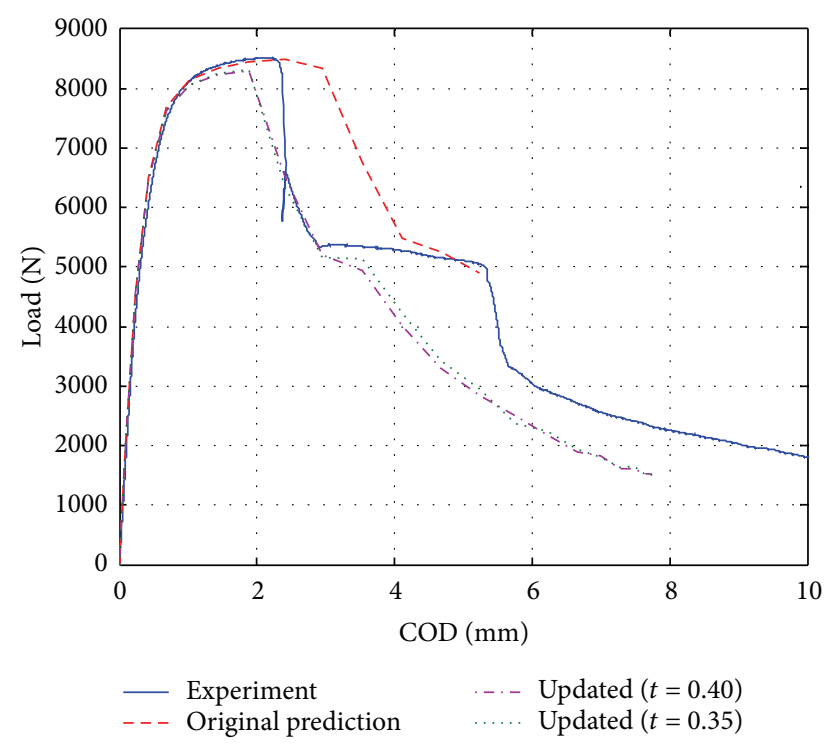

FIGURE 8: S5 load versus COD profiles from experiment and simulations with varying stress triaxiality thresholds, $t$.

with measurements. Two load versus COD profiles from this methodology are given in Figure 8. The shape of the predicted profiles beyond the first load drop was a marked improvement over the first iteration's; however, the ligament D-C still failed prematurely. This methodology is insufficient. In one regard, damage needs to be induced in order for the Gurson model to predict accurately the first load drop, but damage should not be allowed to evolve as quickly (with respect to load application) in ligament D-C as it did in this scheme. This motivates the use of a shear-modified Gurson model where damage is not induced as invasively as in this methodology.

\section{Discussion}

5.1. Overview. The modeling effort was divided into two phases. During the first phase, the computational model was created, verified, and used to make the aforementioned blind predictions. During the second phase, as-manufactured digital twin specimens were considered to resolve the crackpath ambiguity. Additionally, the model was updated to account for the unexpected shear failure mode in specimens which failed along A-D-C-E. The activities conducted during both phases, summarized in the spiral development model in Figure 9, mimic several of the procedures within Digital Twin. This paper is focused primarily on the shift made to the as-manufactured specimen geometry at the beginning of the second phase, but for the purpose of contextualizing this adoption of higher fidelity geometric models, both phases are discussed here. Note that cross-references to Figure 9 are made via braces, \{\} , hereafter.

5.2. Phase 1. At the onset of the first phase $\{a\}$, the prominence of the Gurson model in previous ductile fracture studies $[15,16]$ was noted. Moreover, given the time constraints of the challenge, the Gurson model, already implemented in Abaqus/Explicit, offered a relatively quick and simple means to estimate Sandia's QOIs. Therefore, it was adopted for this challenge $\{b\}$. It is well known that the Gurson model performs poorly for shear-dominated regimes unless modified [13]; however, no modifications were made. Additionally, some anisotropy was observed in the reported uniaxial tension test results, but the implementation in Abaqus/Explicit only accommodates linear isotropic elasticity, and thus anisotropy was ignored.

There were several sources of uncertainty in the model that should have been considered during the first phase. These included the following.

Geometry. An engineering drawing of the challenge specimen with nominal tolerances was provided by Sandia. These tolerances were ignored as the framework was conditioned on a single nonrandom (nominal) geometry. However, modeling a distribution of specimen geometries within the specified tolerance would not have been informative and the predicted crack path would have always been A-C-E. In other words, without modeling the as-manufactured geometry, accurate predictions could not have been made, further motivating the need for the Digital Twin framework.

Loading Conditions. By design, Sandia reported relatively vague information about loading conditions to mimic real world engineering problems. Clearly, this is a source of uncertainty, but no effort was made to quantify it.

Material Parameters. Sandia provided limited standardized experimental data to calibrate the Gurson model. Some of the model's parameters were able to be calibrated with these data; however, some could not be calibrated and thus were asserted.

Physical QOIs. Physical QOIs were available only in the second phase, as the SFC was to be double-blind.

A mesh refinement study was conducted to determine a characteristic element length $(\sim 200 \mu \mathrm{m})$ such that the crack path was insensitive to the discretization and subsequent refinement yielded negligible change in the solution. As part of the computational model validation, a calibration of the Gurson model parameters was also conducted against tensile test and sharp-crack Mode-I fracture data provided by Sandia. There are four issues to consider with this validation activity. First, neither the tensile nor fracture toughness specimens had shear-mode damage. Consequently, shear failure was ignored during calibration. In hindsight, regardless of whether shear failure was initially hypothesized to occur or not, this omission handicapped calibration efforts. Second, only some of the Gurson model's parameters were able to be calibrated with the provided data. For example, yield surface parameters $q_{1}, q_{2}$, and $q_{3}$ were all specified to be unity in the first phase as no data at the time were available to suggest otherwise. Third, the variability in the calibration data provided was not quantified. Finally, the validation was incomplete in the first phase due to the lack of a physical QOI or a response metric for the challenge specimen. It is noteworthy that incomplete information is a part of most 


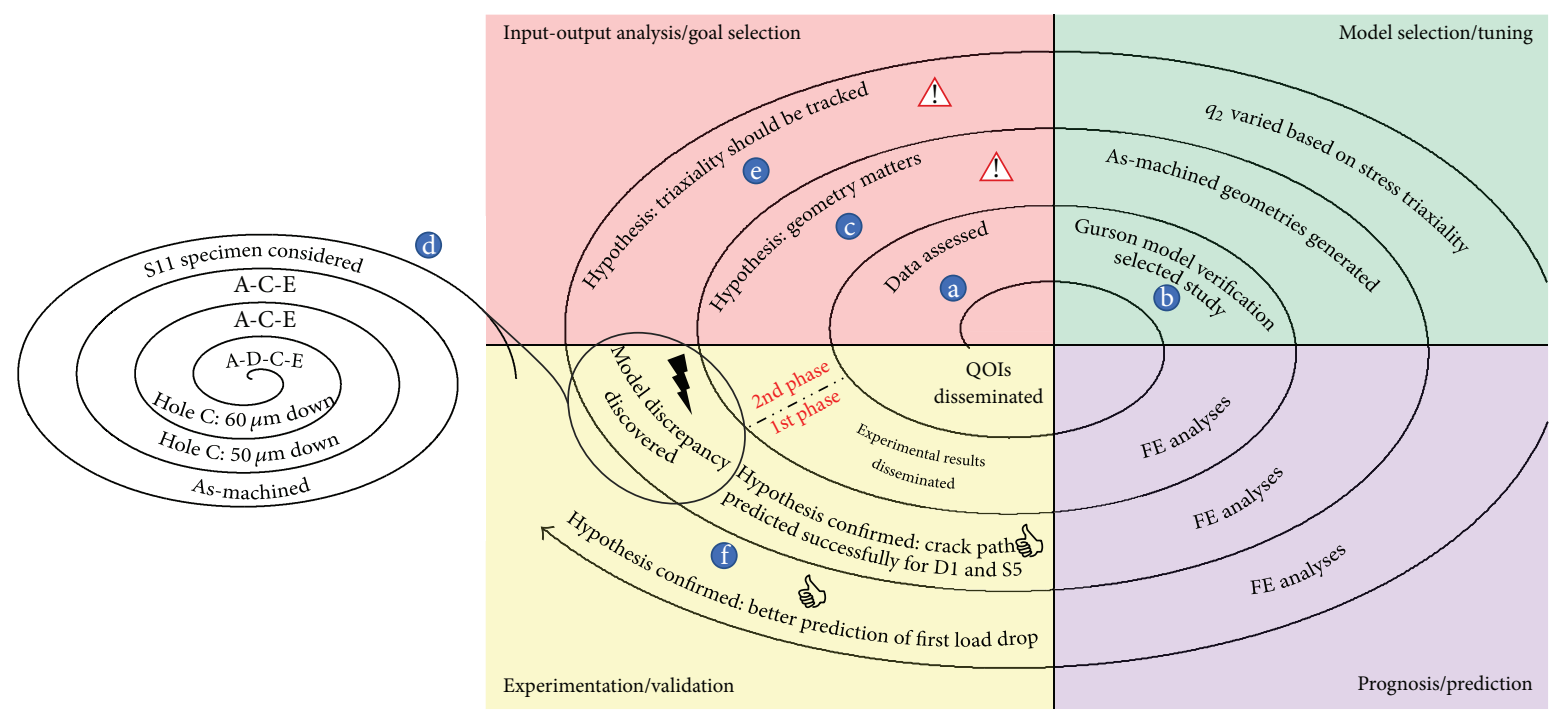

FIGURE 9: Spiral development diagram of both phases of the SFC.

engineering applications. One goal of Digital Twin is to identify the effect of such gaps while a vehicle is in-service and provide a means of filling them via Bayesian updating and model improvements.

5.3. Phase 2. Due to the adoption of as-manufactured component geometry $\{c\}$, the framework was able to predict crack paths for specimens machined to specifications and those exhibiting the highest deviations from specifications; however, it faltered for the S11 specimen which exhibited the subtlest deviations from specifications. This specimen closely resembled geometries that failed $\mathrm{A}-\mathrm{C}-\mathrm{E}$ but cracked along $\mathrm{A}-$ $\mathrm{D}-\mathrm{C}-\mathrm{E}$. The model predicted A-C-E-a model discrepancy that was later bounded to be only $60 \mu \mathrm{m}$ in one specified dimension $\{\mathrm{d}\}$.

Prior to being updated in the second phase, the computational model predicted peak load accurately but could not capture the immediacy of the first load drop in specimens that failed along A-D-C-E. To address this issue, the model was updated to induce damage at material points with low stress triaxiality $\{\mathrm{e}\}$, thereby compensating for the Gurson model's poor performance in shear-dominated regimes. The result was a markedly better prediction of the S5 specimen's first load drop $\{f\}$.

\section{Conclusion}

The SFC is a cautionary tale to the notion that modeling ductile fracture accurately in a nonstandardized geometry can be done with nothing but a representative geometry, a seemingly well-calibrated constitutive model, and sound engineering judgment. The following can be concluded from the 2012 Sandia Fracture Challenge and the associated computational effort presented herein.

(i) The challenge specimen did not have a single representative geometry. Depending on the relative locations of the holes beyond the blunt notch, the challenge specimen was likely to fail along one of two possible paths. (ii) The computational model used during the first phase of the challenge was predicated on the challenge specimen with nominal dimensions (a geometry that never existed and one that was characteristic of only one failure path). Consequently, this model lacked the fidelity to resolve the crack path ambiguity.

(iii) Improved (physics-based) material models should be incorporated into future studies if Bayesian updating as related to unexpected damage modes is to be done.

Digital Twin, a paradigm that could potentially supplant current structural life maintenance and prediction practices, was conceived to maintain the highest level of personalization in fleet management, thereby circumventing ambiguities like the one detailed herein. Although only a few of the requisite activities of Digital Twin were performed in this paper, namely consideration of as-manufactured component geometry, its effectiveness and applicability to even smallscale scenarios such as the SFC are apparent. By applying Digital Twin concepts, the crack-path ambiguity diagnosis signaled a need to consider as-manufactured specimens. Asmanufactured (digital twin) specimens were consequently implemented, predictions were made, and a second round of modeling was conducted to test the effectiveness of the computational model at determining the physical QOIs. Inaccurate predictions were somewhat remedied by compensating for the Gurson model's limitations under shear loading.

In the spirit of Digital Twin, the challenge specimen can be interpreted as a component. A fleet manager who procured several of these challenge specimen components, in this case Sandia National Labs, expected them to behave similarly to one another; however, when subjected to identical loading conditions, they clearly did not. Furthermore, the resulting variation in behavior was not random, but predictable upon consideration of as-manufactured geometry and material model improvements. Most specimens exhibited an A-D-CE crack path while others failed A-C-E. Specimens with the same crack path exhibited a significant spread in their load 
versus COD profiles, an observation that can be attributed primarily to geometric and secondarily to material-scale variations. Future research on this topic should inquire whether each specimen's place in this distribution can be determined and used to further personalize a component's digital twin. Consequently, the need to consider each challenge specimen as a part endowed with its own behavioral characteristics was essential in the SFC. Within the Digital Twin paradigm, components are not commoditized, they are personalized.

\section{Nomenclature}

$\begin{array}{ll}E: & \text { Young's modulus } \\ f: & \text { Void volume fraction } \\ f_{c}: & \text { Critical void volume fraction } \\ f_{f}: & \text { Void volume fraction at total failure } \\ f_{N}: & \text { Volume fraction of nucleated voids } \\ J_{3}: & \text { Third invariant of the deviatoric stress tensor } \\ q_{1}, q_{2}, q_{3}: & \text { yield surface parameters } \\ r: & \text { Relative density } \\ s_{N}: & \text { Standard deviation of nucleation strain } \\ t: & \text { Stress triaxiality below which damage is induced } \\ \varepsilon_{N}: & \text { Mean nucleation strain } \\ v: & \text { Poisson's ratio } \\ \sigma_{e}: & \text { Effective Mises stress } \\ \sigma_{M}: & \text { Yield stress of fully dense matrix } \\ \sigma_{k}^{k}: & 3 \times \text { (hydrostatic stress). }\end{array}$

\section{Conflict of Interests}

The authors declare that there is no conflict of interests regarding the publication of this paper.

\section{Acknowledgments}

The authors would like to acknowledge Dr. Brad Boyce of Sandia National Labs for spearheading the 2012 Sandia Fracture Challenge as well as all of its participants. Andrew Gross and Professor Ravi-Chandar of the University of Texas at Austin are also acknowledged for providing the experimental data of the S11 specimen that appear in this paper. Dr. Aida Nonn of Salzgitter Mannesmann Forschung $\mathrm{GmbH}$ is also recognized for her assistance with the porous metal plasticity model. Jacob Hochhalter would like to acknowledge support from the NASA Aeronautics Research Institute Seedling Fund which provided the necessary resources to complete the simulation work presented. This research was made possible by support from the Ross-Tetelman Fellowship at Cornell University.

\section{References}

[1] L. Christodoulou and J. M. Larsen, "Using materials prognosis to maximize the utilization potential of complex mechanical systems," JOM, vol. 56, no. 3, pp. 15-19, 2004.

[2] E. H. Glaessgen and D. S. Stargel, “The Digital Twin paradigm for future NASA and U.S. Air Force vehicles," in Proceedings of the 53rd AIAA/ASME/ASCE/AHS/ASC Structures, Structural
Dynamics and Materials Conference, Honolulu, Hawaii, USA, April 2012.

[3] E. J. Tuegel, A. R. Ingraffea, T. G. Eason, and S. M. Spottswood, "Reengineering aircraft structural life prediction using a Digital Twin,” International Journal of Aerospace Engineering, vol. 2011, Article ID 154798, 14 pages, 2011.

[4] B. L. Boyce, S. L. B. Kramer, H. E. Fang et al., "The Sandia Fracture Challenge: blind round robin predictions of ductile tearing," International Journal of Fracture, vol. 186, pp. 5-68, 2014.

[5] A. J. Gross and K. Ravi-Chandar, "Prediction of ductile failure using a local strain-to-failure criterion," International Journal of Fracture, vol. 186, no. 1-2, pp. 69-91, 2014.

[6] K. Nahshon, M. Miraglia, J. Cruce, R. DeFrese, and E. T. Moyer, "Prediction of the Sandia Fracture Challenge using a shear modified porous plasticity model," International Journal of Fracture, vol. 186, pp. 93-105, 2013.

[7] K. Pack, M. Luo, and T. Wierzbicki, "Sandia Fracture Challenge: blind prediction and full calibration to enhance fracture predictability," International Journal of Fracture, vol. 186, no. 1-2, pp. 155-175, 2014.

[8] T. Zhang, E. Fang, P. Liu, and J. Lua, "Modeling and simulation of 2012 Sandia Fracture Challenge problem: phantom paired shell for Abaqus and plane strain core approach," International Journal of Fracture, vol. 186, pp. 117-139, 2013.

[9] Simulia, Abaqus 6.11 User Manual, Simulia, Providence, RI, USA, 2011.

[10] A. L. Gurson, "Continuum theory of ductile rupture by void nucleation and growth : part I-yield criteria and flow rules for porous ductile media," Journal of Engineering Materials and Technology, vol. 99, no. 1, pp. 2-15, 1977.

[11] V. Tvergaard, "Influence of voids on shear band instabilities under plane strain conditions," International Journal of Fracture, vol. 17, no. 4, pp. 389-407, 1981.

[12] R. Courant, K. Friedrichs, and H. Lewy, "On the partial difference equations of mathematical physics," IBM: Journal of Research and Development, vol. 11, pp. 215-234, 1967.

[13] K. Nahshon and J. W. Hutchinson, "Modification of the Gurson Model for shear failure," European Journal of Mechanics, A/Solids, vol. 27, no. 1, pp. 1-17, 2008.

[14] O. Chabanet, D. Steglich, J. Besson, V. Heitmann, D. Hellmann, and W. Brocks, "Predicting crack growth resistance of aluminium sheets," Computational Materials Science, vol. 26, pp. $1-12,2003$.

[15] V. Tvergaard and A. Needleman, "Analysis of the cup-cone fracture in a round tensile bar," Acta Metallurgica, vol. 32, no. 1, pp. 157-169, 1984.

[16] W. Brocks, D. Klingbeil, G. Kunecke, and D-Z. Sun, "Application of the Gurson Model to ductile tearing resistance," in Constraint Effects in Fracture. Theory and Applications, vol. 2, pp. 232-254, ASTM, Philadelphia, Pa, USA, 1995. 

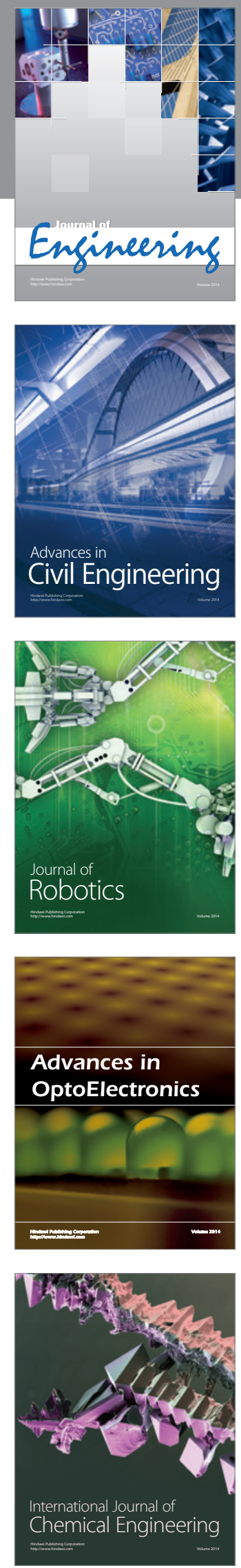

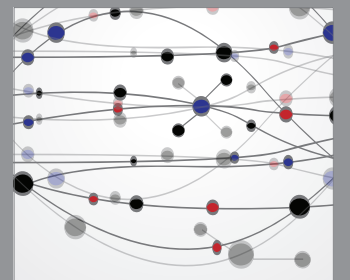

The Scientific World Journal
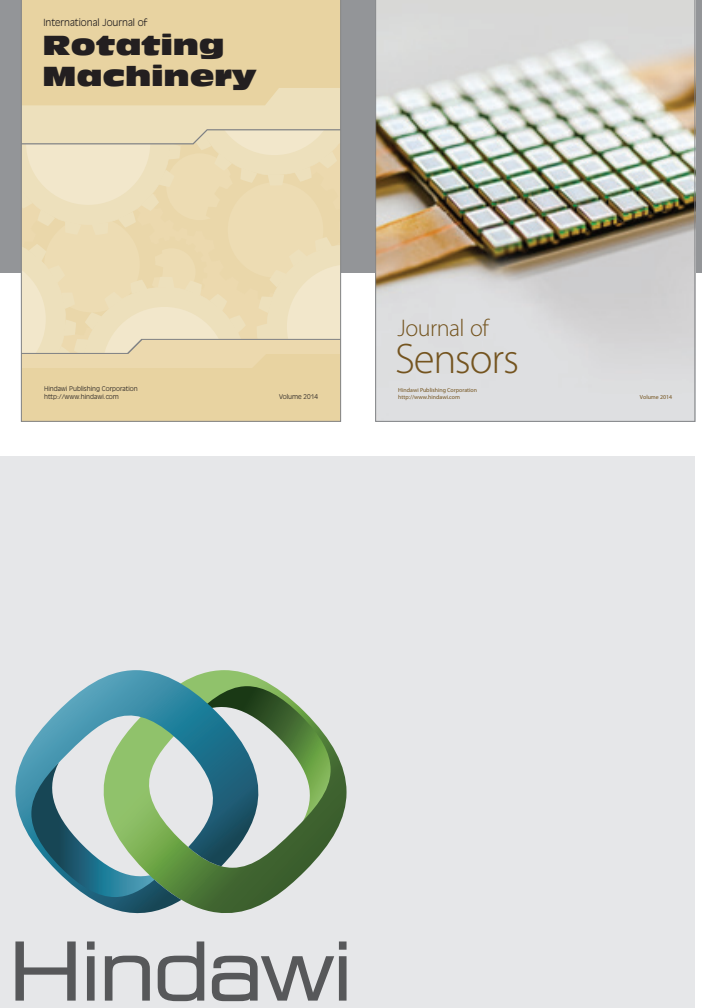

Submit your manuscripts at http://www.hindawi.com
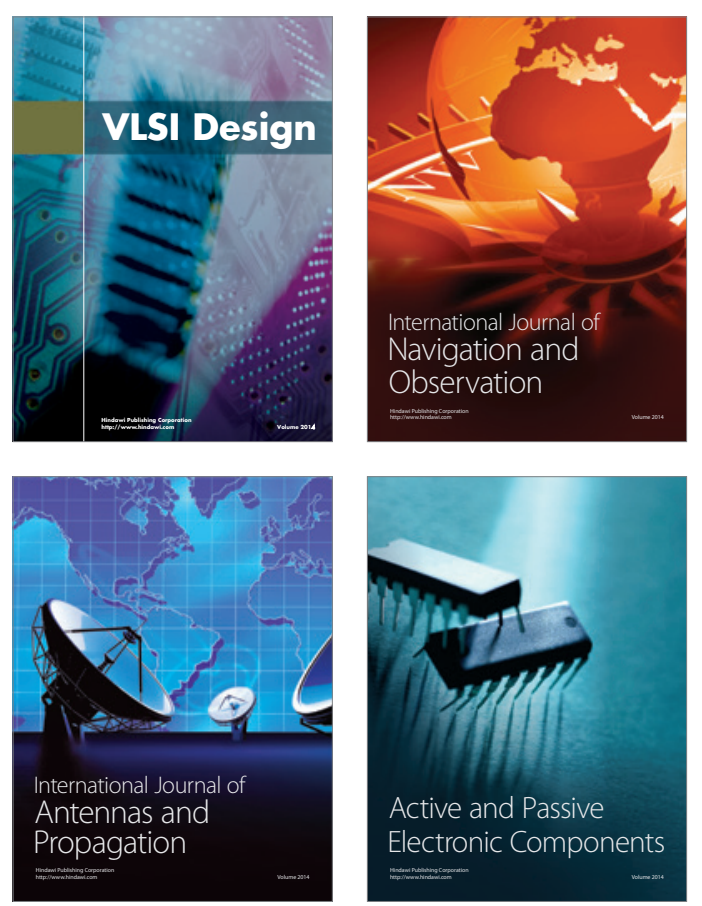
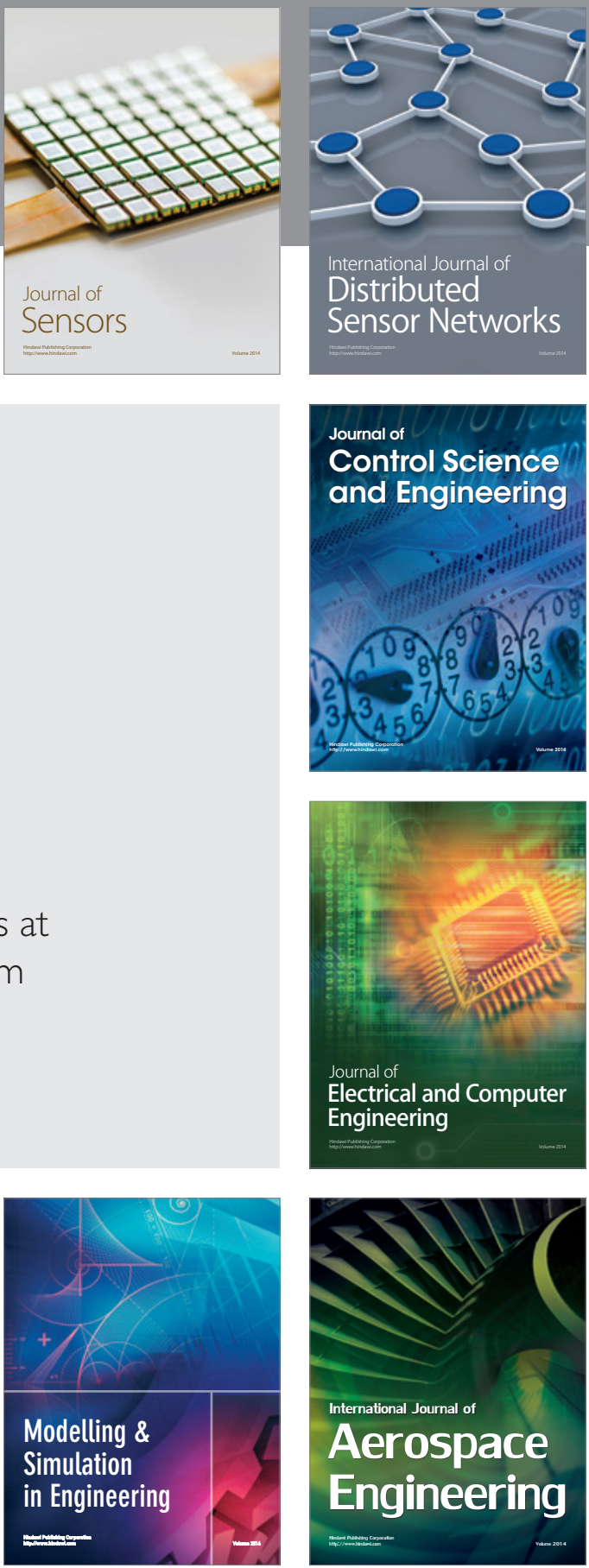

Journal of

Control Science

and Engineering
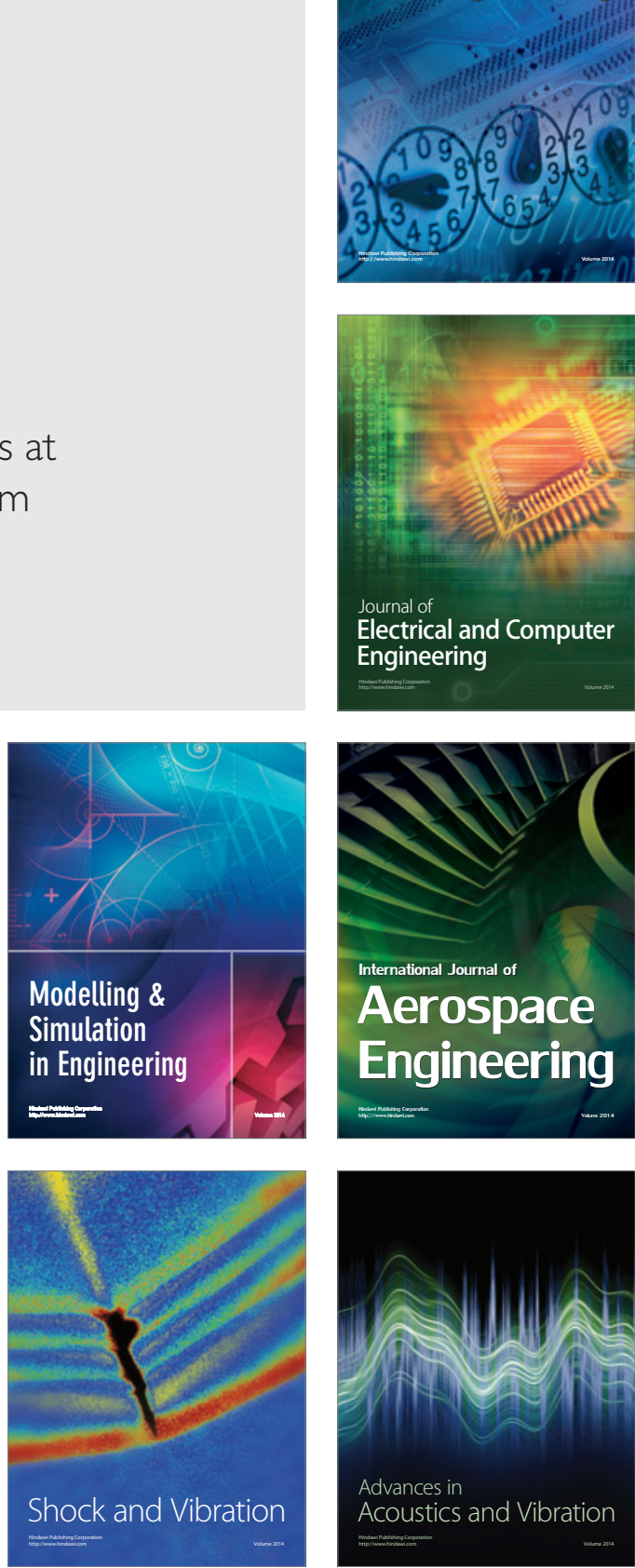